\title{
Sterol-resistant transcription in CHO cells caused by gene rearrangement that truncates SREBP-2
}

\author{
Jianxin Yang, Ryuichiro Sato, Joseph L. Goldstein, and Michael S. Brown \\ Department of Molecular Genetics, University of Texas Southwestern Medical Center at Dallas, Dallas, Texas 75235 USA
}

\begin{abstract}
Sterol-resistant CHO cells (SRD-1 cells) fail to repress sterol synthesis and LDL receptor gene transcription when incubated with 25-hydroxycholesterol. Here we trace the defect to a rearrangement in the gene encoding SREBP-2, a membrane-bound transcription factor that regulates cholesterol homeostasis. SREBP-2 is an 1139-amino acid protein that is bound to extranuclear membranes via a carboxy-terminal attachment domain. In sterol-depleted cells a protease liberates the amino-terminal fragment ( $\sim 480$ amino acids). This fragment, which contains the transcriptional activation and bHLH-Zip domains, translocates to the nucleus. 25-Hydroxycholesterol abolishes protease activity and halts transcription. SRD-1 cells produce a soluble, truncated form of SREBP-2 (amino acids 1-460) that lacks the membrane attachment domain and activates transcription directly, bypassing the sterol-regulated proteolytic step. Although SRD-1 cells produce full-length SREBP-2 from the wild-type allele and a related transcription factor, SREBP-1, they fail to cleave both of these precursors, indicating that the truncated form of SREBP-2 down-regulates the protease through a form of end-product feedback inhibition. The current data provide genetic evidence for the previously proposed model in which cholesterol homeostasis is controlled by sterol-regulated proteolysis of a membrane-bound bHLH-Zip transcription factor.
\end{abstract}

[Key Words: Cholesterol; 25-hydroxycholesterol-resistant CHO cells; sterol-regulated gene transcription; bHLH-Zip transcription factors]

Received June 8, 1994; accepted July 7, 1994.

Two sterol-regulatory element binding proteins, designated SREBP-1 and SREBP-2, control cholesterol homeostasis in animal cells (Hua et al. 1993; Yokoyama et al. 1993; Wang et al. 1994). When cells are deprived of sterols, active fragments of both proteins enter the nucleus and bind to a sterol regulatory element, designated SRE1 , in the 5'-flanking region of the genes for 3-hydroxy-3methylglutaryl CoA (HMG CoA) synthase and the low density lipoprotein (LDL) receptor. Binding leads to transcriptional activation, and this in turn leads to enhanced cholesterol synthesis as well as to enhanced uptake of cholesterol from plasma LDL. When cholesterol accumulates, the active forms of both SREBPs disappear from the nucleus, and transcription declines, thereby preventing overaccumulation of cholesterol by the cell.

A novel proteolytic mechanism controls the activities of both SREBPs (Sato et al. 1994; Wang et al. 1994). SREBP- 1 is synthesized as a $125-\mathrm{kD}$ precursor that is embedded in the membranes of the endoplasmic reticulum (ER) and nuclear envelope. The amino-terminal segment of SREBP-1 ( $\sim 480$ amino acids) contains an acidic transcriptional activation domain and a basic-helix-loophelix-leucine zipper (bHLH-Zip) region that mediates protein dimerization and DNA binding. The carboxy-terminal segment of SREBP-1 ( $\sim 660$ amino acids) contains two long hydrophobic sequences that are postulated to span the membranes of the ER and nuclear envelope. In the absence of sterols, a protease cleaves the protein between the bHLH-Zip region and the transmembrane domains, releasing an amino-terminal fragment of $\sim 480$ residues that travels to the nucleus and activates transcription. When sterols accumulate within cells, the activity of the protease diminishes, and the active fragment is no longer generated (Sato et al. 1994; Wang et al. 1994).

Human SREBP-2 is $47 \%$ identical to human SREBP-1 (Hua et al. 1993). Both contain acidic amino-terminal sequences, bHLH-Zip regions, and putative transmembrane domains. Either protein can activate transcription from SRE-1 containing promoters when overexpressed in cells by transfection. Each protein appears capable of acting independently, and there is no evidence of functional heterodimerization (Hua et al. 1993). Both proteins appear to be regulated by proteolysis in a similar fashion (Wang et al. 1994; J. Yang, R. Sato, J. Goldstein, and M. Brown, unpubl.). The cDNA for hamster SREBP-1 has also been cloned, and its deduced amino acid sequence shows $81 \%$ identity to that of human SREBP-1 (Sato et al. 1994).

If the SREBPs normally regulate transcription from SRE-1 containing promoters, then abnormalities in SREBP should be found in mutant cells that have defects 
in cholesterol homeostasis. Several laboratories have isolated sterol-resistant mutant cell lines that fail to repress transcription of genes controlling enzymes of the sterol biosynthetic pathway and the LDL receptor when incubated with sterols (Chen et al. 1979; Sinensky et al. 1979; Chang and Chang 1982; Peffley et al. 1988; Metherall et al. 1989; Dawson et al. 1991). Our laboratory reported three independent isolates of sterol-resistant defective cells, which we designated SRD-1, SRD-2, and SRD-3 (Metherall et al. 1989; Dawson et al. 1991). These cells were isolated after treatment of Chinese hamster cells with nitrosoethyl urea (SRD-1 cells), $\gamma$-irradiation (SRD-2), or ethyl methanesulfonate (SRD-3). To select for sterol resistance, the mutagenized hamster cells were grown in culture medium devoid of lipoproteins and supplemented with 25-hydroxycholesterol. Under these conditions, wild-type cells die because 25-hydroxycholesterol suppresses cholesterol synthesis, but it cannot replace cholesterol in the cell membrane. Mutant cells that have lost the ability to repress cholesterol biosynthesis survive in the presence of 25-hydroxycholesterol.

Although the SRD-1, SRD-2, and SRD-3 cells were selected solely for resistance to repression of sterol biosynthesis, the cells also were found to be resistant to transcriptional suppression of the LDL receptor gene by 25 hydroxycholesterol (Metherall et al. 1989; Dawson et al. 1991). The cells had no defect in the uptake of 25 -hydroxycholesterol as evidenced by the observation that the sterol retained the ability to stimulate cholesterol ester synthesis and to accelerate the proteolytic degradation of HMG CoA reductase, two actions that do not involve transcriptional control (Dawson et al. 1991). In transfection studies, SRD-1 cells were shown to be more active than parental Chinese hamster ovary $(\mathrm{CHO})$ cells in transcribing genes driven by synthetic or natural promoters that contain the SRE-1 sequence (Metherall et al. 1989|. Elevated transcription required an intact SRE-1 sequence in the promoter. In contrast to normal cells, transcription was not reduced in the SRD-1 cells when 25-hydroxycholesterol was added. We postulated that the SRD-1 cells contain a constitutive factor that activates transcription by binding to SRE-1 and is not repressed by sterols. The newly discovered SREBPs are likely candidates.

In the current studies, we report that SRD-1 cells do indeed have a mutation in the gene for one of the SREBPs, namely, SREBP-2. These cells produce a truncated form of SREBP-2 that lacks the membrane spanning regions and thus does not require proteolysis to enter the nucleus and activate gene transcription. The observation that $\mathrm{CHO}$ cells use a truncated form of SREBP-2 to escape sterol-mediated regulation provides strong evidence in support of the sterol regulated-cleavage model for transcriptional control of cholesterol homeostasis.

\section{Results}

cDNA cloning of mutant SREBP-2

Figure 1 shows the amino acid sequence and domain structure of hamster SREBP-2 as predicted from the sequence of a cDNA isolated from SRD-1 cells. Screening cDNA libraries from these cells yielded two types of clones, one of which encoded full-length SREBP-2 (1139 amino acids) and the other of which encoded a truncated version of the protein (see below). The full-length protein showed an overall $93 \%$ identity with human SREBP-2. The resemblance extended to all of the domains noted previously in human SREBP-2, including the amino-terminal acidic region, which is believed to be a transcriptional activating domain, the bHLH-Zip region, and the two putative transmembrane regions (residues $480-500$ and 532-552, respectively).

In addition to the cDNA clones encoding wild-type SREBP-2, the cDNA library from the SRD-1 cells yielded a shorter cDNA that contains the $5^{\prime}$ end of the SREBP-2 transcript fused to a portion of a transcript derived from another gene (Fig. 2). This cDNA encodes the aminoterminal portion of SREBP-2, terminating at residue 460 , after which the sequence diverges from that of the wildtype SREBP-2 cDNA. The divergent sequence shows a high level of identity with the $3^{\prime}$ sequence of a mouse cDNA, reported previously, encoding a protein termed the Ku p70 autoantigen (Porges et al. 1990). The nucleotide identities with the mouse $\mathrm{Ku}$ p70 antigen cDNA are indicated by the asterisks in Figure 2. The reading frame of the hamster SREBP-2 sequence does not align with the reading frame of the hamster $\mathrm{Ku}$ p 70 antigen. As a result, the $\mathrm{Ku}$ p 70 antigen cDNA encodes 11 novel amino acids that are not found in the $\mathrm{Ku}$ p 70 protein followed by a stop codon. The mutant fusion protein is predicted to contain 471 amino acids, of which the amino-terminal 460 residues are identical to the aminoterminal portion of SREBP-2 and the carboxy-terminal 11 amino acids are novel. This sequence includes the acidic transcriptional activation domain and the bHLHZip DNA binding domain of SREBP-2, but it eliminates the transmembrane domains and the long carboxy-terminal extension.

\section{Truncated SREBP-2 results from a gene rearrangement}

Figure 3 shows a Northern blot analysis of RNA isolated from wild-type CHO-7 cells and mutant SRD-1 cells. When probed with the SREBP-2 specific oligonucleotides, the wild-type cells showed a predominant mRNA of $\sim 5 \mathrm{~kb}$. There was also a small amount of a $4.3-\mathrm{kb}$ mRNA, which may represent an alternative site of polyadenylation. The SRD-1 cells contained the normallength mRNA, and in addition they contained a $1.9-\mathrm{kb}$ mRNA that was approximately the same size as the aberrant cDNA that was cloned from these cells. The mRNA for SREBP-1 was of normal length and amount in the SRD-1 cells (Fig. 3).

To confirm that the 1.9-kb SREBP-2 mRNA in the SRD-1 cells corresponded to the aberrant cDNA clone, we probed Northern blots with ${ }^{32} \mathrm{P}$-labeled oligonucleotides corresponding to the portion of the mutant SREBP-2 cDNA derived from the Ku p70 antigen gene. Wild-type CHO-7 cells exhibited a faint band with these 
Figure 1. Comparison of the amino acid sequences and domain structures of hamster and human SREBP-2. (TOp) Dashed lines denote residues in human SREBP-2 that are identical to hamster SREBP-2. The bHLH-Zip region is boxed. The two overlines denote hydrophobic stretches of amino acids. DNA polymorphisms were identified at nucleotide positions 1638 (A) $\mathrm{G}$ ) and $1682(\mathrm{~T} / \mathrm{C})$ (for reference, nucleotide 159 is the A of the first initiation codon). Half of the cDNAs contained $\mathrm{A}$ at 1638 and $T$ at 1682; the other half contained $\mathrm{G}$ and $\mathrm{C}$, respectively. The nucleotide 1638 polymorphism causes an amino acid change from asparagine to serine at amino acid 493 as denoted by the asterisk $\left({ }^{*}\right)$. The other polymorphism caused no amino acid substitution. The full-length nucleotide sequences of hamster pSREBP-2 and human pSREBP-2 are deposited in GenBank (accession number U12330 and U0203, respectively). (Bottom) Domain structures of hamster and human SREBP-2 with numbers corresponding to the amino acids. The bHLH-Zip region is denoted by the black box and the carboxy-terminal domain by the shaded box. The two putative transmembrane domains are indicated. Regions enriched in particular amino acids are indicated. The \% identities between the hamster and human sequences are indicated.
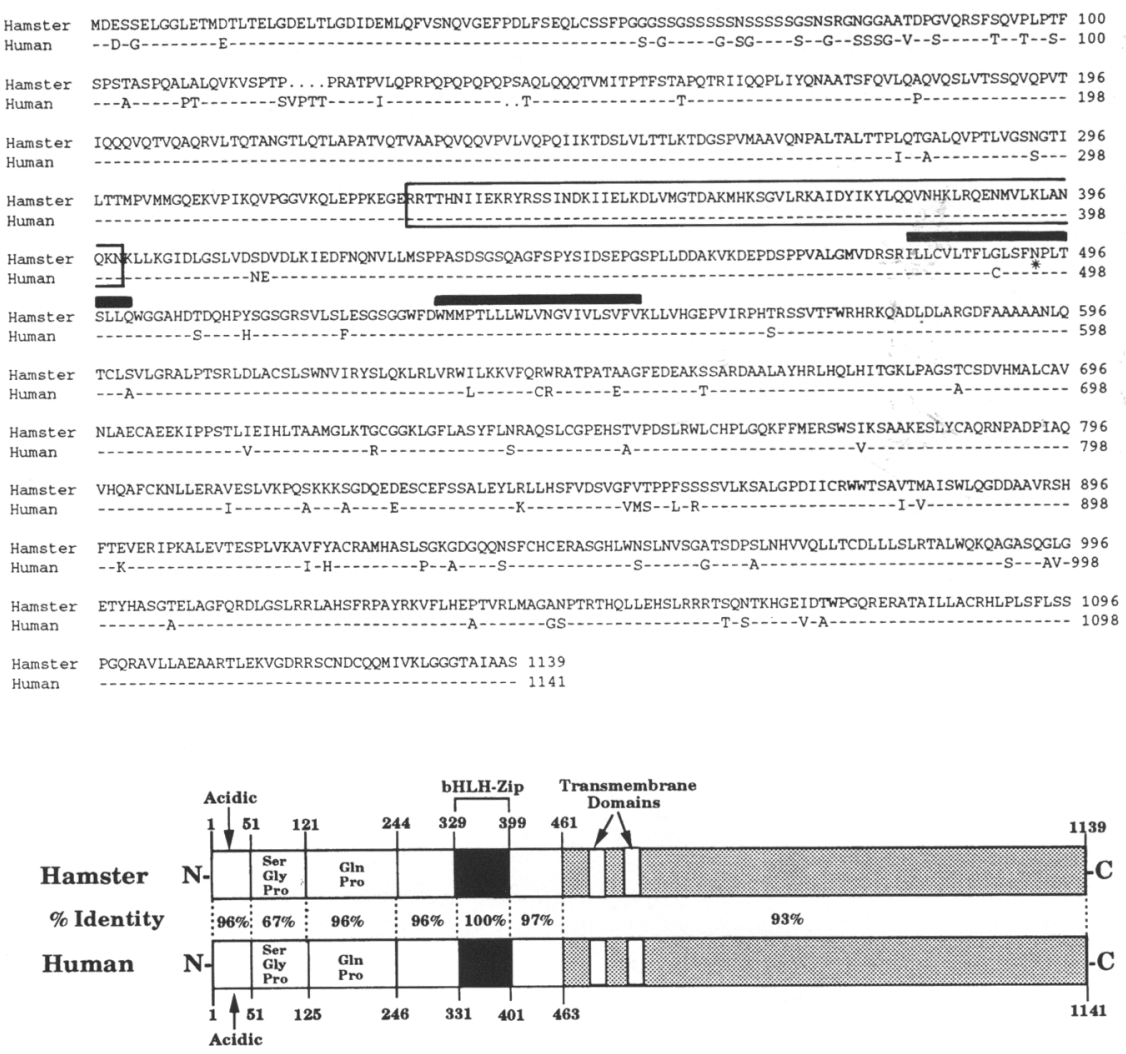

probes (Fig. 4). The size of this mRNA corresponds to the size of the mouse Ku p70 antigen cDNA reported previously (Porges et al. 1990). In the SRD-1 cells, the Ku p70 antigen probes revealed an additional band at $1.9 \mathrm{~kb}$, which is the same size as the mRNA detected with the SREBP-2 probes (see Fig. 3).

The data of Figures 2-4 suggest that SRD-1 cells have undergone a genomic rearrangement that brings the $5^{\prime}$ end of the SREBP-2 gene into proximity with the $3^{\prime}$ end of the Ku p70 antigen gene. To detect such a rearrangement, we carried out Southern blot analyses of genomic DNA from CHO-7 and SRD-1 cells probed with the cDNAs for SREBP-2 and the Ku p70 antigen. Figure 5 shows that the SRD-1 cells contained novel genomic fragments (indicated by solid dots) that were present after digestion with PstI or StuI and were revealed by the SREBP-2 probe. Similarly, the Ku p70 probe hybridized to aberrant bands when the SRD-1 DNA was digested with BamHI, EcoRI, or HindIII. These data indicate that the SREBP-2 gene and the $\mathrm{Ku}$ p 70 gene have both undergone rearrangements in the SRD-1 cells.

Although we performed extensive digestion with multiple restriction enzymes, we were unable to find an enzyme that produced a single aberrant genomic fragment that hybridized with both the Ku p70 antigen cDNA probe and the SREBP-2 cDNA probe. We believe that this is because the fusion between the two genes occurs within a large intron. Indeed, PCR analysis of genomic DNA from CHO-7 cells suggests that an intron of $>1 \mathrm{~kb}$ splits the coding region of SREBP-2 at a position corresponding approximately to amino acid 460 . So far, all of the restriction enzymes that have been tested appear to cut within this intron, thereby separating the SREBP-2 fragment from the $\mathrm{Ku}$ p70 fragment.

\section{Truncated SREBP-2 down regulates proteolytic cleavage of wild-type SREBP-2 and SREBP-1}

The predicted size of the SREBP-2/Ku p70 fusion protein is slightly smaller than the active nuclear fragment of SREBP-2 that is released from cell membranes by sterolregulated proteolysis (Wang et al. 1994). In contrast to the wild-type proteolytic product, the fusion protein should be produced in the presence as well as the absence of sterols. To test this hypothesis, we performed an immunoblot analysis using an antibody against SREBP-2 in extracts of $\mathrm{CHO}-7$ and SRD-1 cells that were grown under various conditions (Fig. 6A). The immunoblots were performed with a $100,000 \mathrm{~g}$ pellet of cell membranes (upper) and with a high salt nuclear extract (lower). As reported previously for SREBP-1 (Wang et al. 1994), CHO-7 cells grown in the absence of sterols showed a $\sim 125-\mathrm{kD}$ membrane-bound precursor form of SREBP-2 (upper, lane 1) and a $\sim 68-\mathrm{kD}$ nuclear fragment 

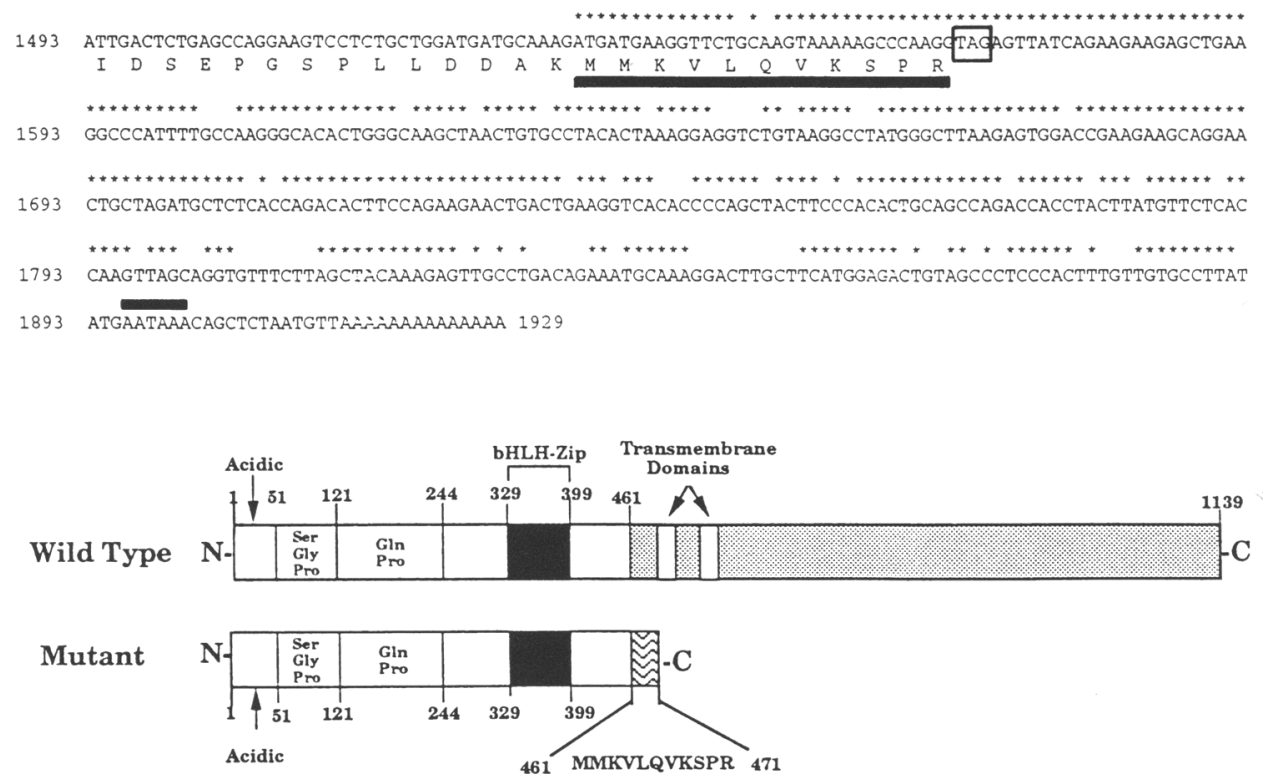

Figure 2. Structure of mutant SREBP-2 cDNA and protein in SRD-1 cells. (Top) Nucleotide sequence of the cDNA encoding the mutant form of SREBP-2 in the region where the $5^{\prime}$ end of SREBP-2 is fused to the $3^{\prime}$ end of hamster Ku p70 autoantigen. The nucleotide sequence of residues 1-1492 is identical to that of the wild-type hamster SREBP-2 cDNA and is not shown. Stars denote nucleotides that are identical to the sequence of mouse Ku p70 cDNA (Porges et al. 1990). The mutant cDNA encodes a protein of 471 amino acids. The first 460 amino acids are identical to wild-type SREBP-2 and the last 11 (denoted by the underline) are encoded by the Ku p70 autoantigen cDNA. The stop codon is boxed. The overline denotes the polyadenylation signal sequence. (Bottom) Domain structures of wild-type and mutant SREBP-2. The numbers correspond to the amino acid sequence. The 11 unique amino acids encoded by the hamster Ku p70 autoantigen cDNA are shown. Other features of the domain map are as described in the legend to Figure 1. The full-length nucleotide sequence of the mutant SREBP-2 from SRD-1 cells is deposited in GenBank (accession number U12329).

(lower, lane 1). Addition of sterols led to a disappearance of the $68-\mathrm{kD}$ mature form in the nuclear extract (lane 2), whereas addition of the cholesterol synthesis inhibitor compactin had the opposite effect increasing the proteolytic form in the nuclear extract (lane 3). We showed previously that the cysteine protease inhibitor $\mathrm{N}$-acetylleucyl-leucyl-norleucinal (ALLN) blocks the degradation of the nuclear form of SREBP-1 (Wang et al. 1994). This inhibitor also blocks the degradation of the nuclear form of SREBP-2, causing it to accumulate within the nucleus (Fig. 6A, lane 4). The inhibitor also increased the amount of the membrane-bound form of SREBP-2 (lane 4). In the presence of ALLN as in its absence, sterols lowered the amount of the nuclear form of SREBP-2 (lane 5).

In the SRD-1 cells, a truncated nuclear form of SREBP-2 /which consistently migrated slightly faster than the normal nuclear form) was present in the absence of sterols (Fig. 6A, lane 7), and it was not decreased by sterol treatment (lane 8) nor was there an increase when compactin was added (lane 9). In the presence of ALLN, the amount of the truncated nuclear form increased dramatically in the SRD-1 cells, and again there was no regulation by sterols (lanes 10-12). In four experiments, we consistently failed to observe a band that corresponded to the normal mature form of SREBP-2 in the SRD-1 cells grown either in the absence or presence of sterols (lanes 7-12).

We also performed an immunoblot analysis using an antibody against SREBP-1. The antibody visualized the precursor form of SREBP-1 as a doublet at 125 and 115 $\mathrm{kD}$ (upper, Fig. 6B). The nuclear form was seen as a cluster of bands in the $60-$ to $68-\mathrm{kD}$ region. In wild-type CHO-7 cells, the nuclear form of this protein was suppressed by sterols (lane 2) and induced by compactin (lane 3). Similar results were obtained in the presence of ALLN (lanes 4-6). In striking contrast, the SRD-1 cells showed no evidence of a mature form of SREBP-1 (lower, lanes 7-12), although they contained abundant amounts of the membrane-bound precursor form (upper, lanes 7-12). We attribute this finding to a suppression of the activity of the SREBP cleavage enzyme, owing to the constitutive expression of the truncated form of SREBP-2 in these cells.

Suppression of the SREBP cleavage enzyme in SRD-1 cells may result from end-product feedback, owing to the overproduction of cholesterol. To test this possibility, we incubated SRD-1 cells and control CHO-7 cells in lipoprotein-deficient serum with a high concentration of compactin, an inhibitor of cholesterol synthesis, for periods up to $72 \mathrm{hr}$ (Fig. 7), at which point toxicity is observed from sterol depletion. To confirm that the cells were depleted of cholesterol, we measured the cell's ability to incorporate $\left[{ }^{14} \mathrm{C}\right]$ oleate into cholesteryl$\left[{ }^{14} \mathrm{C}\right]$ oleate, a reaction that is proportional to the cellular pool of free cholesterol (Goldstein et al. 1983). When grown in lipoprotein-deficient serum, the SRD-1 cells 


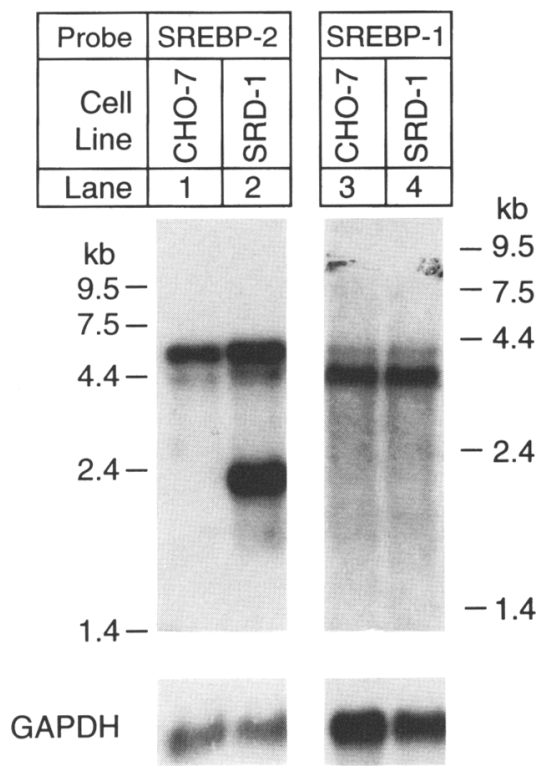

Figure 3. Northern blot analysis of SREBP-2 and SREBP-1 mRNA in CHO-7 and SRD- 1 cells. An aliquot of poly $(\mathrm{A})^{+}$RNA $(2 \mu \mathrm{g} /$ lane $)$ from the indicated cell line was subjected to agarose gel electrophoresis, transferred to nylon membranes, and hybridized with the indicated ${ }^{32} \mathrm{P}$-labeled probe. (Lanes 1 and 2) Hybridization was carried out with a mixture of three ${ }^{32} \mathrm{P}$-labeled oligonucleotide probes (a total of $4 \times 10^{6} \mathrm{cpm} / \mathrm{ml}$ ) corresponding to nucleotides $85-131,137-184$, and 191-235 of the p02JY9 (SREBP-2) sequence. The hybridization was performed at $50^{\circ} \mathrm{C}$ for $16 \mathrm{hr}$ as described in Materials and methods. The filter was exposed at $-80^{\circ} \mathrm{C}$ for 3 days. The same filter was stripped at $100^{\circ} \mathrm{C}$ for $20 \mathrm{~min}$ in $0.1 \times$ SSC and $1 \%$ SDS and reprobed with a ${ }^{32} \mathrm{P}$-labeled cDNA probe for rat glyceraldehyde3-phosphate dehydrogenase (GAPDH) $\left(2 \times 10^{6} \mathrm{cpm} / \mathrm{ml}\right)$ (Yokoyama et al. 1993) and was exposed at $-80^{\circ} \mathrm{C}$ for $5 \mathrm{hr}$. (Lanes 3 and 4) Hybridization was carried out with a ${ }^{32} \mathrm{P}$-labeled full-length cDNA probe of hamster SREBP-1 $\left(4 \times 10^{6} \mathrm{cpm} / \mathrm{ml}\right)$ (Sato et al. 1994) at $42^{\circ} \mathrm{C}$ for $16 \mathrm{hr}$ as described in Materials and methods. The filter was exposed at $-80^{\circ} \mathrm{C}$ for 3 days. The same filter was stripped and reprobed with the ${ }^{32} \mathrm{P}$-GAPDH probe as described above and exposed at $-80^{\circ} \mathrm{C}$ for $5 \mathrm{hr}$.

had a 10-fold higher rate of cholesteryl ester synthesis than did the CHO-7 cells (zero time, 7C,D). In both cell types, the rate of esterification declined by $90 \%$ over 72 hr. As expected, compactin increased the amount of mature SREBP-2 (A) and SREBP-1 (B) in CHO-7 cells. Despite their apparent cholesterol depletion, the SRD-1 cells showed no trace of the mature form of either SREBP-2 (A) or SREBP-1 (B) after incubation with compactin for times up to $72 \mathrm{hr}$.

\section{Truncated SREBP-2 stimulates transcription of sterol-regulated genes}

To confirm that the truncated form of SREBP-2 is active in stimulating transcription of sterol-regulated genes, we prepared an expression plasmid encoding the mutant protein and introduced it into embryonic kidney 293 cells by transfection simultaneously with plasmids encoding chloramphenicol acetyltransferase (CAT) under control of promoter sequences from the LDL receptor, HMG CoA synthase, and HMG CoA reductase genes. The truncated SREBP-2 was more active than wild-type SREBP-2 in stimulating transcription from the promoter containing tandem copies of repeats 2 and 3 from the LDL receptor gene (Fig. 8A). Repeat 2 contains the SRE-1 sequence that is a target of SREBP, and repeat 3 contains an $\mathrm{Spl}$ site that is required for transcription (Briggs et al. 1993). Similar results were seen with the promoter containing the $5^{\prime}$ flanking region of the HMG CoA synthase gene (Fig. 8B). However, no stimulation of the HMG CoA reductase promoter was observed (Fig. $8 \mathrm{C}$ ). These results correspond to results with wild-type SREBP-1 reported previously (Yokoyama et al. 1993) and SREBP-2 (Hua et al. 1993).

\section{Discussion}

Although sterol-resistant cell lines were first isolated $>15$ years ago (Chen et al. 1979; Sinensky et al. 1979; Dawson et al. 1991), the molecular defects that underlie this phenotype have been unknown. In the current paper, we provide evidence that the defect in one of these

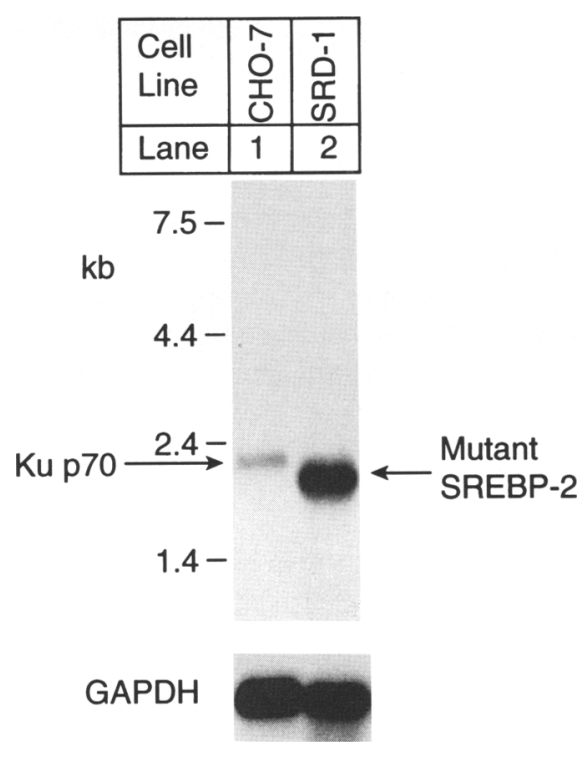

Figure 4. Northern blot analysis of poly $(A)^{+}$RNA from CHO-7 and SRD-1 cells with $\mathrm{Ku}$ p70 autoantigen probes. Poly $(\mathrm{A})^{+}$RNA preparation, gel-electrophoresis, and hybridization were performed as described in Materials and methods and in the legend to Figure 3. The probes used (upper) are a mixture of two ${ }^{32} \mathrm{P}$-labeled $43 \mathrm{mer}$ oligonucleotides (total of $4 \times 10^{6} \mathrm{cpm}$ / $\mathrm{ml}$ ), which are specific to the portion of mutant SREBP-2 that corresponds to the sequence of the cDNA encoding hamster Ku p70 autoantigen (nucleotides 1547-1579 and 1683-1725 of p02JY7 in Fig. 2). The filter was exposed at $-80^{\circ} \mathrm{C}$ for 3 days. The same filter was stripped and reprobed with $\left[{ }^{32} \mathrm{P}\right] \mathrm{GAPDH}$ (lower) as described in the legend to Fig. 3 and was exposed at $-80^{\circ} \mathrm{C}$ for $5 \mathrm{hr}$. 


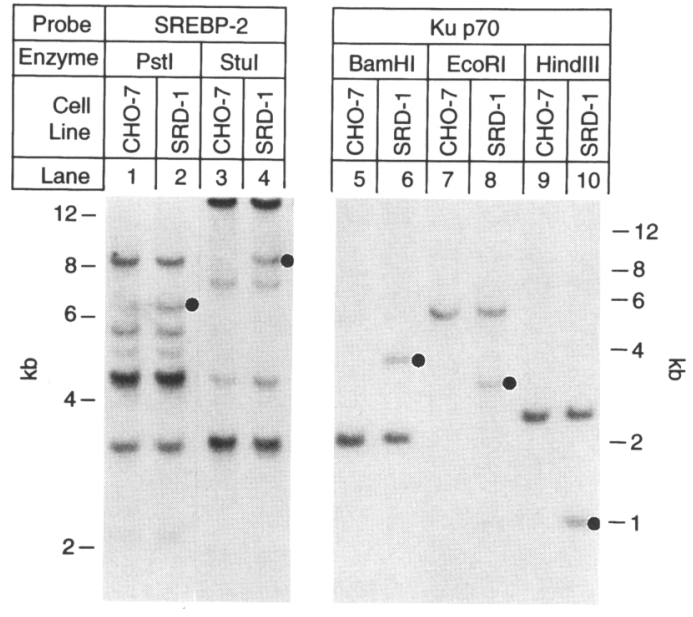

Figure 5. Southern analysis of SREBP-2 and $\mathrm{Ku}$ p70 autoantigen genes in CHO-7 and SRD-1 cells. Aliquots of genomic DNA (10 $\mu \mathrm{g} /$ lane) from the indicated cell line were digested with the indicated restriction enzyme, subjected to electrophoresis on $0.8 \%$ agarose gels, transferred to nylon membranes, and hybridized with the indicated ${ }^{32}$ P-labeled probe. (Lanes 1-4) Hybridization was carried out with a ${ }^{32} \mathrm{P}$-labeled full-length SREBP-2 cDNA probe $\left(4 \times 10^{6} \mathrm{cpm} / \mathrm{ml}\right)$ at $42^{\circ} \mathrm{C}$ for $20 \mathrm{hr}$ as described in Materials and methods. The filter was exposed at $-80^{\circ} \mathrm{C}$ for 3 days. (Lanes 5-10) Hybridization was carried out with a ${ }^{32} \mathrm{P}$ labeled cDNA probe $\left(4 \times 10^{6} \mathrm{cpm} / \mathrm{ml}\right)$ corresponding to the $\mathrm{Ku}$ p70 autoantigen sequence of mutant SREBP-2 (nucleotide 15381815 of p02JY7 in Fig. 2). The filter was exposed at $-80^{\circ} \mathrm{C}$ for 4 days. In lanes $1-10$, the abnormal bands in SRD-1 cells are indicated by dots.

cell lines, SRD-1 cells, is a gene rearrangement that produces an mRNA encoding a truncated form of SREBP-2 that acts autonomously, bypassing the requirement for sterol-regulated proteolysis. This finding solves at least one of the mysteries underlying the sterol-resistant phenotype, and it also provides strong and independent ev- idence that the SREBPs are physiologic regulators of the transcription of the HMG CoA synthase and LDL receptor genes.

The aberrant mRNA in SRD-1 cells contains sequences derived from the $5^{\prime}$ and $3^{\prime}$ ends of the SREBP-2 and $\mathrm{Ku}$ p70 autoantigen genes, respectively. Rearrangements in both of these genes were documented by the Southern blots in Figure 5. The exact rearrangement that brought these two genes into juxtaposition is unknown. If the SREBP- 2 gene and the Ku p70 autoantigen gene are normally adjacent, then the rearrangement might be a deletion that removes the 3 ' end of the SREBP- 2 gene and the $5^{\prime}$ end of the Ku p70 autoantigen gene. If the two genes are normally on different chromosomes, then the genetic rearrangement must involve a translocation that unites the genes on a single chromosome.

In addition to the cDNA encoding truncated SREBP-2, we isolated two different cDNAs encoding full-length SREBP-2 from the SRD-1 cells. These cDNAs differed from each other at two nucleotides in the coding region (see legend to Fig. 1). Using a reverse transcription/PCR method, we demonstrated that the parental $\mathrm{CHO}$ cells produce both of the full-length mRNAs in approximately a 1:1 molar ratio (data not shown). We therefore believe that these two sequences represent a genetic polymorphism and that the parental CHO cells are heterozygous at this locus. The SRD-1 cells must therefore contain at least 3 copies of the SREBP-2 gene, that is, two fulllength copies plus the mutant copy. This raises the possibility that the mutant form of SREBP-2 arises from a partial duplication. Alternatively, the cells may exhibit a triploidy for the region of the chromosome that normally contains SREBP-2.

The truncated protein produced in the SRD-1 cells contains the amino-terminal 460 amino acids of SREBP- 2 followed by 11 amino acids that are produced from the $\mathrm{Ku} \mathrm{p} 70$ gene in an alternate reading frame. These 11 amino acids are not normally found in the $\mathrm{Ku}$ p70 antigen (Porges et al. 1990; Reeves and Sthoeger
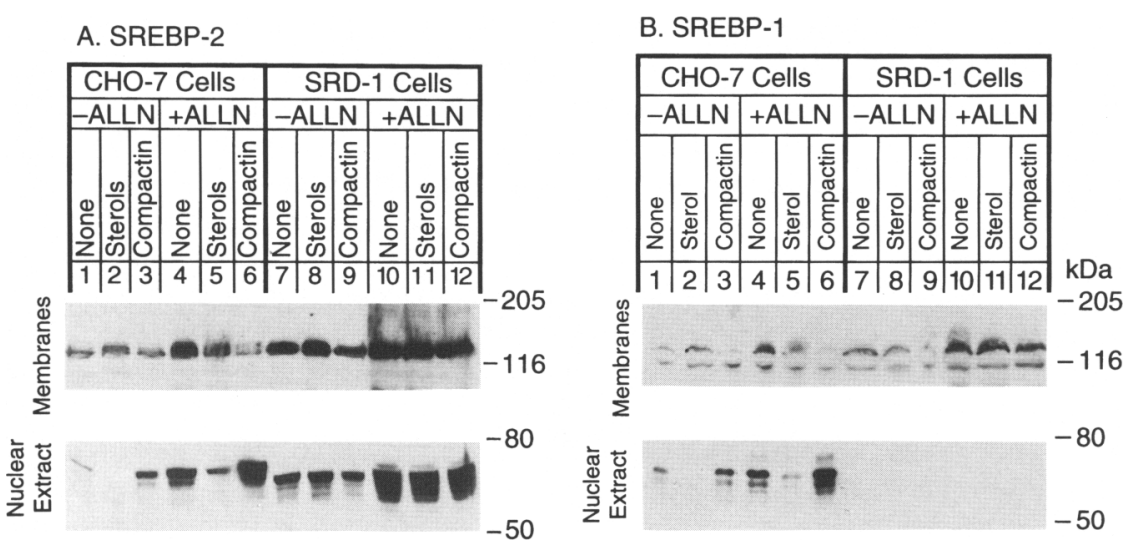

Figure 6. Immunoblot analysis of SREBP- $2(A)$ and SREBP- $1(B)$ in CHO-7 and SRD-1 cells. On day 0 , the indicated cells were set up at $5 \times 10^{5}$ cells per $100 \mathrm{~mm}$ dish in medium A. On day 2 , cells in lanes 3,6 , 9 , and 12 were refed with fresh medium A containing $100 \mu \mathrm{M}$ compactin and $100 \mu \mathrm{M}$ sodium mevalonate. Cells in all other lanes received only medium A. On day 3 , the following additions were made directly to the dish: (lanes 2, 5, 8, and 11) $1 \mu \mathrm{g} 25$ hydroxycholesterol plus $10 \mu \mathrm{g} / \mathrm{ml}$ cholesterol; (lanes 4-6 and 10-12) $100 \mu \mathrm{g} / \mathrm{ml}$ ALLN. The media in all dishes were adjusted to contain $0.2 \%$ (vol/vol) ethanol and $0.2 \%$ (vol/vol) DMSO, the solvents for sterols and ALLN, respectively. After incubation for $4 \mathrm{hr}$ at $37^{\circ} \mathrm{C}$, the cells were harvested, and the $100,000 \mathrm{~g}$ membrane fractions $($ top $)$ and high salt nuclear extracts (bottom) were prepared as described in Materials and methods. An aliquot of each fraction (50 $\mu \mathrm{g}$ protein) was subjected to SDS-PAGE and immunoblot analysis with either anti-SREBP-2 antibody J911 (1:1000 dilution of antiserum) ( $A$ ) or 4 $\mu \mathrm{g} / \mathrm{ml}$ of IgG-2A4, a monoclonal antibody directed against SREBP-1 $(B)$. The gels were calibrated with Prestained SDS-PAGE Standards High Range (Bio Rad). The filters were exposed to film for $30 \mathrm{sec}$. 
A. SREBP-2

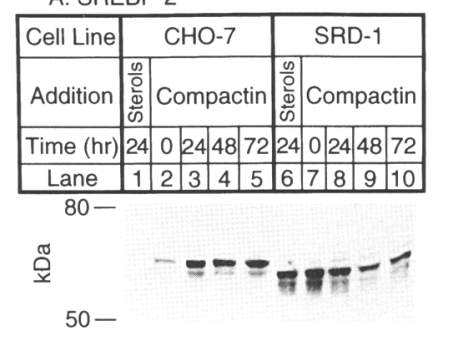

B. SREBP-1

\begin{tabular}{|c|c|c|c|}
\hline \multicolumn{2}{|r|}{ CHO-7 } & \multicolumn{2}{|r|}{ SRD-1 } \\
\hline $\begin{array}{l}\frac{\omega}{0} \\
\frac{0}{\infty} \\
\text { के }\end{array}$ & Compactir & & Compactin \\
\hline 24 & \begin{tabular}{|l|l|l|l|}
0 & 24 & 48 & 7 \\
\end{tabular} & & \begin{tabular}{|l|l|l|l|l|}
0 & 24 & 48 & 72 \\
\end{tabular} \\
\hline 1 & \begin{tabular}{|l|l|l|l}
2 & 3 & 4 & 5 \\
\end{tabular} & 6 & \begin{tabular}{|l|l|l|l|}
7 & 8 & 9 & 10 \\
\end{tabular} \\
\hline
\end{tabular}

$-80$

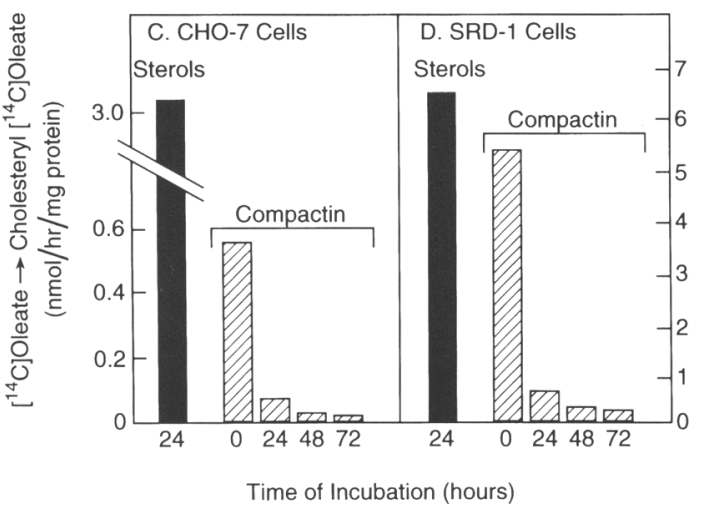

Figure 7. Compactin does not stimulate proteolysis of SREBP-2 $(A)$ or SREBP-1 $(B)$ in SRD-1 cells. On day 0, CHO-7 and SRD-1 cells were set up at $3.5 \times 10^{5}$ cells per $100-\mathrm{mm}$ dish in medium A. On days 1, 2, and 3 cells were refed with medium A containing either compactin plus $50 \mu \mathrm{M}$ sodium mevalonate (lanes 2-5 and 7-10 in $A$ and $B$; striped bars in $C$ and $D$ ) or 1 $\mu \mathrm{g} / \mathrm{ml}$ 25-hydroxycholesterol plus $10 \mu \mathrm{g} / \mathrm{ml}$ cholesterol (lanes 1 and 5 in $A$ and $B$; closed bars in $C$ and $D)$ and incubated at $37^{\circ} \mathrm{C}$ for the indicated time. $(A$ and $B)$ On day 4 , cells were harvested, and high salt nuclear extracts were prepared as described in Materials and methods. Immunoblot analysis of SREBP-1 and 2 in the nuclear extracts was carried out as described in the legend to Figure 6. The filters were exposed to film for $20 \mathrm{sec}$. $\mid C$ and $D \mid$ On day 4, cells were pulsed-labeled for $2 \mathrm{hr}$ with $0.1 \mathrm{~mm}$ $\left[{ }^{14} \mathrm{C}\right]$ oleate-albumin $(8012 \mathrm{dpm} / \mathrm{nmole})$, after which the cells were harvested for measurement of their cholesteryl $\left[{ }^{14} \mathrm{C}\right]$ oleate content (Goldstein et al. 1983). Each value is the average of triplicate incubations.

1989|, and they represent a novel sequence that is not likely to contribute to the sterol-resistant phenotype.

In parental $\mathrm{CHO}$ cells the active amino-terminal fragment of SREBP-2 was present only in the absence of sterols (Fig. 6A). In the SRD-1 cells the truncated aminoterminal fragment of SREBP-2 was present when the cells were grown in the presence as well as in the absence of 25-hydroxycholesterol (Fig. 6A). This result indicates that the mutant fragment is synthesized constitutively through translation of the aberrant mRNA, thus bypassing the requirement for sterol-regulated proteolysis in order to reach the nucleus and activate transcription.

Transfection experiments confirmed that the truncated SREBP-2 is capable of stimulating transcription driven by SRE-1 sequences within the 5'flanking region of the HMG CoA synthase promoter and a synthetic pro- moter that contains two copies of repeats $2+3$ from the LDL receptor gene. The truncated protein was more active than full-length SREBP-2 in stimulating this transcription (Fig. 8A,B). Similar effects on transcription have been reported with an amino-terminal fragment of SREBP-1 (amino acids 1-476) that was created in vitro by site-directed mutagenesis (Sato et al. 1994).

For technical reasons, the transfection method could not be used to demonstrate directly that the truncated SREBP-2/Ku p70 fusion protein had lost its sensitivity to inhibition by sterols. This is because sterols do not reduce the activity even of wild-type SREBP-1 or SREBP-2 when they have been overexpressed as a result of transfection (Yokoyama et al. 1993; Hua et al. 1993). In transient transfection experiments, the SREBPs are produced at levels that are many times higher than those that are produced in untransfected cells. Under these artificial conditions, small amounts of active fragments may be generated by adventitious proteases that cleave SREBPs inefficiently and only when the proteins are massively overexpressed. Alternatively, under unphysiologic conditions of massive overexpression, some full-length SREBP-1 or -2 may enter the nucleus and activate transcription without a requirement for proteolysis.

In SRD-1 cells, the mRNA for HMG CoA reductase is overproduced several-fold, and it is not suppressed by sterols (Metherall et al. 1989). This finding suggests that truncated SREBP-2 stimulates transcription of the HMG CoA reductase gene. However, we have not been able to demonstrate that overexpression of SREBP-1 or SREBP-2 increases transcription of the CAT gene when it is driven by a promoter containing $509 \mathrm{bp}$ from the $5^{\prime}$ flanking region of the HMG CoA reductase gene (Yokoyama et al. 1993; Hua et al. 1993), even though this promoter fragment contains sequences sufficient for sterol-regulated transcription (Osborne et al. 1988; Metherall et al. 1989). Moreover, the truncated SREBP-2 produced by the aberrant mRNA from the SRD- 1 cells also failed to stimulate transcription driven by this promoter construct (Fig. 7C). This HMG CoA reductase promoter fragment does not contain a sequence that corresponds precisely to the consensus sequence for DNA binding by SREBP-1 or SREBP-2 (Wang et al. 1993; Osborne 1991). It is possible that the native HMG CoA reductase gene contains a sterol regulatory element that is activated by SREBPs, but not present in the fragment that we have used for transfection.

An important observation in the current studies is the failure of the SRD-1 cells to produce detectable amounts of the active forms of SREBP-1 or SREBP-2, even when the cells were deprived of sterols and were grown in the presence of the cholesterol synthesis inhibitor compactin (Figs. 6 and 7). This finding suggests that SREBP-1 and SREBP-2 may both be cleaved by a single sterolregulated protease that is down-regulated in response to the constitutive production of the active form of SREBP2. The active amino-terminal fragment of SREBP-2 might suppress proteolysis by inhibiting the protease or repressing the gene that encodes it. Alternatively, the mutant SREBP-2 fragment might induce or activate a 

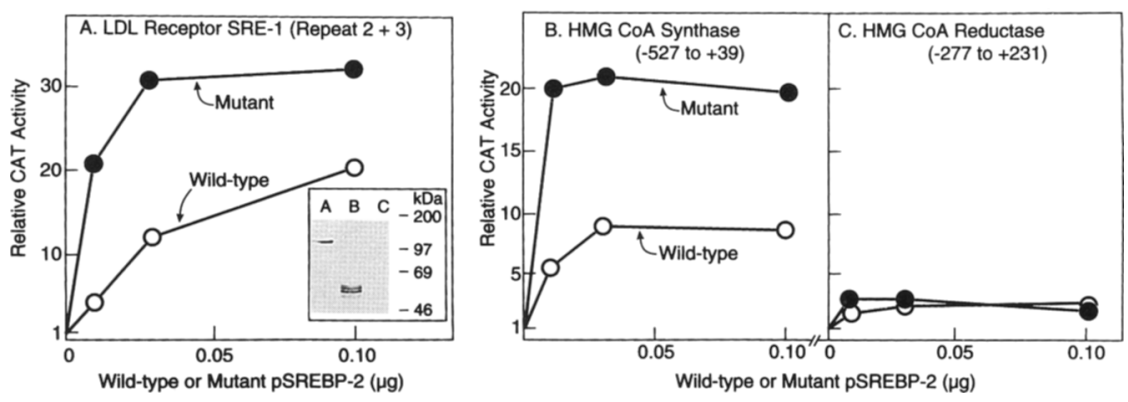

Figure 8. Effect of wild-type and mutant SREBP-2 on CAT activity under control of promoters for LDL receptor $(A)$, HMG CoA synthase $(B)$, and $H M G$ CoA reductase $(C)$ in transfected 293 cells. (A) 293 cells were cotransfected with varying amounts of the indicated wild-type or mutant pSREBP-2 plasmid and $1 \mu \mathrm{g}$ of a promoter-CAT reporter gene driven by a synthetic DNA sequence containing two tandem copies of repeat $2+3$ from the LDL receptor promoter (plasmid K). ( $B$ and C) 293 cells were cotransfected with varying amounts of the indicated wild-type or mutant pSREBP-2 plasmid and a promoter-CAT reporter gene driven by one of the following native promoter elements: $(B)$ HMG CoA synthase, nucleotides -527 to $+39(1 \mu \mathrm{g}$ of plasmid J); or $(C) \mathrm{HMG}$ CoA reductase, nucleotides -277 to $+231(1 \mu \mathrm{g}$ of pRedCAT-1). After $48 \mathrm{hr}$, duplicate dishes of cells were harvested for measurement of CAT activity. Each value is an average of data from two transfection experiments. Relative CAT activity was calculated by dividing the measured CAT activity by the CAT activity in cells cotransfected with a control pRc/CMV7SB vector. The average values for CAT control activity were $421 \mathrm{pmol} / \mathrm{min} / \mathrm{mg}$ protein $(A), 654(B)$, and $1568(C)$. (Inset in $A)$ Immunoblot analysis (20 $\mu \mathrm{g}$ protein/lane) of whole cell extracts of 293 cells transfected with $0.3 \mu \mathrm{g}$ of one of the following plasmids: pSREBP-2 (lane A), mutant pSREBP-2 (lane $B$ ), or control vector pRc/CMV7SB (lane C). After electrophoresis on a $7.5 \%$ polyacrylamide gel in the presence of SDS, the proteins were subjected to immunoblot analysis with $5 \mu \mathrm{g} / \mathrm{ml} \mathrm{IgG-j911} \mathrm{as} \mathrm{described} \mathrm{in} \mathrm{Materials} \mathrm{and} \mathrm{methods.} \mathrm{The} \mathrm{filter} \mathrm{was} \mathrm{exposed} \mathrm{to} \mathrm{film}$ for $30 \mathrm{sec}$.

protein that inhibits the proteolysis. This would be a classic form of end-product feedback inhibition inasmuch as the amino-terminal fragment of SREBP-2 is a product of the protease.

In previous studies we concluded that the phenotype of the SRD-1 cells is genetically recessive to the wildtype (Dawson et al. 1991). However, the production of a truncated, nonregulated form of SREBP-2 should disrupt cholesterol homeostasis in a genetically dominant fashion. The previous conclusion was reached on the basis of cell hybridization experiments using SRD-1 cells and parental $\mathrm{CHO}$ cells that were rendered resistant to the antibiotics G-418 and hygromycin. After fusion with polyethylene glycol, lines of hybrid cells were isolated by growth in the presence of both antibiotics. The hybrid cells were sensitive to killing by 25 -hydroxycholesterol, indicating that the parental phenotype was dominant. As controls, we showed that homotypic fusions between G-418 resistant and hygromycin-resistant SRD-1 cells produced hybrids that retained the 25 -hydroxycholesterol-resistant phenotype. In the light of the present data, the most likely explanation for the previous finding is the loss of the single copy of the mutant chromosome from the SRD-1/parental CHO hybrid cells, which were grown for many generations prior to testing for 25-hydroxycholesterol sensitivity. Cells produced from homotypic fusions between SRD-1 cells must lose two copies of the mutant chromosome to revert to the parental phenotype, an event that is statistically less likely than the loss of a single mutant chromosome. These observations illustrate a potential pitfall in tests of genetic dominance produced by somatic cell hybridization.

To date, we have studied the actions of SREBP-1 and SREBP-2 only on the promoters for HMG CoA synthase, HMG CoA reductase, and the LDL receptor. It is likely, however, that these transcription factors act on promoters for genes encoding other enzymes in the cholesterol biosynthetic pathway /Goldstein and Brown 1990; Chang and Chang 1982). The factors may also influence genes encoding enzymes in fatty acid metabolism as evidenced by the observation that fatty acid metabolism is altered in certain 25-hydroxycholesterol-resistant cell lines (Panini et al. 1990).

Efforts are under way to determine whether defects in SREBP-1 or SREBP- 2 underlie the sterol-resistant phenotype of SRD-2 and SRD-3 cells. In addition, we are exploring the possibility that defects in these proteins are responsible for the failure to activate the genes for the LDL receptor and cholesterol biosynthetic enzymes in sterol-requiring auxotrophs (Chin and Chang 1982; Evans and Metherall 1993).

\section{Materials and methods}

\section{General materials and methods}

Standard molecular biology techniques were used (Sambrook et al. 1989). DNA sequencing was performed with the dideoxy chain termination method on an Applied Biosystems Model 373A DNA sequencer. Newborn calf lipoprotein-deficient serum $(\mathrm{d}>1.215 \mathrm{~g} / \mathrm{ml})$ was prepared by ultracentrifugation (Goldstein et al. 1983). Sterols, calpain inhibitor I (ALLN), and other chemicals were obtained from sources described previously (Wang et al. 1994). Reporter CAT genes (pRedCAT-1 and plasmids $\mathrm{K}$ and $\mathrm{J}$ ) have been described previously (Yokoyama et al. 1993|. Protein was measured with the BCA* Protein Assay Reagent (Pierce). Oligonucleotide probes were ${ }^{32} \mathrm{P}$-labeled by end labeling with polynucleotide kinase, and cDNA probes were ${ }^{32}$ P-labeled with a Random Primed DNA Labeling Kit (Boehringer Mannheim). Restriction enzymes were obtained from New England Biolabs. The incorporation of $\left[{ }^{14} \mathrm{Cloleate-albumin}\right.$ into cholesteryl $\left[{ }^{14} \mathrm{C}\right]$ esters in intact cells was carried out as described previously (Goldstein et al. 1983)

\section{Cell culture}

SRD-1 cells, a mutant line of 25-hydroxycholesterol-resistant 
CHO-7 cells, were isolated as described previously (Metherall et al. 1989|. All cells were grown in monolayer at $37^{\circ} \mathrm{C}$ in an atmosphere of $9 \% \mathrm{CO}_{2}$. CHO-7 cells were maintained in medium A (a 1:1 mixture of Ham's F-12 medium and Dulbecco's modified Eagle's minimum essential medium containing 100 units/ $\mathrm{ml}$ penicillin, $100 \mu \mathrm{g} / \mathrm{ml}$ streptomycin sulfate, $2 \mathrm{~mm}$ glutamine, and $5 \%(\mathrm{vol} / \mathrm{vol})$ newborn calf lipoprotein-deficient serum). SRD- 1 cells were maintained in medium A containing $2 \mu \mathrm{g} / \mathrm{ml}$ 25-hydroxycholesterol.

\section{CDNA cloning of wild-type and mutant hamster SREBP-2}

Poly $(\mathrm{A})^{+}$RNA from SRD-1 cells was isolated with oligo(dT) affinity columns (GIBCO-BRL) and used to construct two cDNA libraries. The synthesized double-stranded cDNA was fractionated on a cDNA Size Fractionation Column (GIBCO-BRL), directionally cloned into $\lambda g t 22 A$ (GIBCO-BRL) and $\lambda D R 2$ vectors (Clontech), and packaged with a $\lambda$ Packaging System (GIBCO$\mathrm{BRL})$. The libraries were screened with the $5^{\prime} 2614-\mathrm{bp}{ }^{32} \mathrm{P}-\mathrm{la}-$ beled fragment of pXH-4, a cDNA clone of human SREBP-2 (Hua et al. 1993), at $30^{\circ} \mathrm{C}$ overnight in $30 \%$ (vol/vol) formamide, $5 \times$ Denhardt's, $100 \mu \mathrm{g} / \mathrm{ml}$ denatured salmon sperm DNA, and $0.1 \%$ (wt/vol) SDS. Among $10^{6}$ plaques screened in the $\lambda g t 22$ library, 10 positive clones were identified. Two of them, p02JY9 and p02JY10, encoded the wild-type hamster SREBP-2 and contained 5003 and $4335 \mathrm{bp}$, respectively. Among $10^{6}$ plaques screened in the $\lambda D R 2$ library, 8 positive clones were identified. One of them, p02JY7, was 1929 bp in length and encoded a mutant form of SREBP-2. All three cDNAs were sequenced on both strands.

\section{Blot hybridization of genomic DNA and poly $(A)^{+}$RNA}

To prepare genomic DNA and total RNA, CHO-7 and SRD-1 cells were cultured in medium A for 3 days before harvesting. Genomic DNA was prepared by ethanol precipitation after proteinase K digestion (Gross-Bellard et al. 1973). For Southern blotting, $10 \mu \mathrm{g}$ of genomic DNA was loaded onto each lane after digestion with the appropriate restriction enzyme. Hybridization with ${ }^{32} \mathrm{P}$-cDNA probes was performed at $42^{\circ} \mathrm{C}$ in $50 \%$ formamide, $5 \times$ SSPE, $10 \times$ Denhardt's, $100 \mu \mathrm{g} / \mathrm{ml}$ salmon sperm DNA, and $2 \%$ (wt $/ \mathrm{vol}$ ) SDS. Total RNA was prepared by ultracentrifugation on cesium chloride after the cells were lysed with guanidine thiocyanate (Sambrook et al. 1989). Poly $\mid \mathrm{A})^{+}$ RNA was isolated with oligo(dT) affinity columns (GIBCOBRL). For Northern blotting, $2 \mu \mathrm{g}$ poly $(\mathrm{A})^{+}$RNA was loaded onto each lane, and hybridization was carried out with ${ }^{32} \mathrm{P}$ labeled oligonucleotide probes at $50^{\circ} \mathrm{C}$ in $10 \times \mathrm{SSPE}, 2 \times$ Denhardt's, $100 \mu \mathrm{g} / \mathrm{ml}$ salmon sperm DNA, and $0.5 \%$ SDS, or with ${ }^{32} \mathrm{P}$-labeled cDNA probes at $42^{\circ} \mathrm{C}$ under conditions described above for Southern blotting. All blots were exposed to DuPont NEF-496 X-ray film at $-80^{\circ} \mathrm{C}$ with two intensifying screens.

\section{Antibodies and immunoblot analysis}

A polyclonal antibody 1911 was produced by immunizing rabbits with a bacterially produced protein encoding 6 consecutive histidines followed by amino acids 32-250 of hamster SREBP-2. The cDNA coding sequences were cloned into a $\mathrm{pQE}-30$ vector and expressed in Escherichia coli, and the protein was purified by $\mathrm{Ni}^{2+}$-Sepharose affinity chromatography as described (Yokoyama et al. 1993). Monoclonal antibody IgG-2A4 against human SREBP-1 has been described previously (Sato et al. 1994).

For immunoblot analysis, a 100,000g membrane fraction and a high salt nuclear extract fraction of SRD-1 and CHO-7 cells were prepared as described by Wang et al. (1994). The Enhanced
Chemiluminescence (ECL) Western Blotting Detection System Kit (Amersham) was used according to the manufacturer's instructions with some modifications (Wang et al. 1994). The blots were exposed to DuPont NEF-496 film at room temperature.

\section{Transfection and reporter CAT assay}

Expression plasmids encoding wild-type hamster SREBP-2 (designated pSREBP-2) and mutant SREBP-2 were constructed by cloning the $5-\mathrm{kb}$ insert from p02JY9 and the $1.9-\mathrm{kb}$ insert from p025JY7, respectively, into the SalI-NotI sites of pRc/CMV7SB (Sato et al. 1994).

Monolayers of human kidney 293 cells were set up on day 0 $\left(3 \times 10^{5}\right.$ cells $/ 60 \mathrm{~mm}$ dish) in medium B (Dulbecco's modified Eagle's medium (DMEM), 100 units/ml penicillin, and $100 \mu \mathrm{g} /$ $\mathrm{ml}$ streptomycin) supplemented with $10 \%$ (vol/vol) newborn calf serum. On day 1 , the cells were cotransfected by the calcium-phosphate method with $0-0.1 \mu \mathrm{g}$ wild-type or mutant pSREBP-2 or control $\mathrm{pRc} / \mathrm{CMV7SB}$ vector, $1 \mu \mathrm{g}$ reporter CAT gene, and $0.3 \mu \mathrm{g} \mathrm{pVA}$ as described (Sato et al. 1994). The total amount of cDNA was adjusted to $3.3 \mu \mathrm{g}$ by addition of either $\mathrm{pRc} / \mathrm{CMV7SB}$ (control vector) or salmon sperm DNA. After incubation for $4 \mathrm{hr}$ at $37^{\circ} \mathrm{C}$, the cells were refed with DMEM containing $10 \%$ calf lipoprotein-deficient serum. After $48 \mathrm{hr}$, CAT activity was assayed by the xylene extraction method (Briggs et al. 1993). The protein content of extracts was measured by the method of Bradford (1976).

\section{Acknowledgments}

We thank our colleagues Xiaodong Wang, Xianxin Hua and Mark Evans for helpful suggestions; Lisa Beatty for invaluable help with the tissue culture experiments; Richard Gibson for antibody production; and Jeffry Cormier and Michelle Laremore for DNA sequencing. This research is supported by grants from the National Institutes of Health (HL-20948) and the Perot Family Foundation. R.S. was the recipient of a National Institutes of Health Fogarty International Research Fellowship (FO5 TW04571).

The publication costs of this article were defrayed in part by payment of page charges. This article must therefore be hereby marked "advertisement" in accordance with 18 USC section 1734 solely to indicate this fact.

\section{References}

Bradford, M.M. 1976. A rapid and sensitive method for the quantitation of microgram quantities of protein utilizing the principle of protein-dye binding. Anal. Biochem. 72: 248254.

Briggs, M.R., C. Yokoyama, X. Wang, M.S. Brown, and J.L. Goldstein. 1993. Nuclear protein that binds sterol regulatory element of LDL receptor promoter I. Identification of the protein and delineation of its target nucleotide sequence. J. Biol. Chem. 268: 14490-14496.

Chang, T.-Y. and C.C.Y. Chang. 1982. Revertants of a Chinese hamster ovary cell mutant resistant to suppression by an analogue of cholesterol: Isolation and partial biochemical characterization. Biochemistry 21: 5316-5323.

Chen, H.W., W.K. Cavenee, and A.A. Kandutsch. 1979. Sterol synthesis in variant Chinese hamster lung cells selected for resistance to 25-hydroxycholesterol. I. Biol. Chem. 254: 715-720.

Chin, J. and T.-Y. Chang. 1982. Further characterization of 
a Chinese hamster ovary cell mutant requiring cholesterol and unsaturated fatty acid for growth. Biochemistry 21: 3196-3202.

Dawson, P.A., J.E. Metherall, N.D. Ridgway, M.S. Brown, and J.L. Goldstein. 1991. Genetic distinction between sterol-mediated transcriptional and posttranscriptional control of 3-hydroxy-3-methylglutaryl coenzyme A reductase. /. Biol. Chem. 266: 9128-9134.

Evans, M.J. and J.E. Metherall. 1993. Loss of transcriptional activation of three sterol-regulated genes in mutant hamster cells. Mol. Cell. Biol. 13: 5175-5185.

Goldstein, J.L. and M.S. Brown. 1990. Regulation of the mevalonate pathway. Nature 343: 425-430.

Goldstein, J.L., S.K. Basu, and M.S. Brown. 1983. Receptor-mediated endocytosis of LDL in cultured cells. Methods Enzy. mol. 98: 24l-260.

Gross-Bellard, M., P. Oudet, and P. Chambon. 1973. Isolation of high-molecular-weight DNA from mammalian cells. Eur. J. Biochem. 36: 32-38.

Hua, X., C. Yokoyama, J. Wu, M.R. Briggs, M.S. Brown, J.L. Goldstein, and X. Wang. 1993. SREBP-2, a second basic-helix-loop-helix-leucine zipper protein that stimulates transcription by binding to a sterol regulatory element. Proc. Natl. Acad. Sci. 90: 11603-11607.

Metherall, J.E., J.L. Goldstein, K.L. Luskey, and M.S. Brown. 1989. Loss of transcriptional repression of three sterol-regulated genes in mutant hamster cells. I. Biol. Chem. 264: 15634-15641.

Osborne, T.F. 1991. Single nucleotide resolution of sterol regulatory region in promoter for 3-hydroxy-3-methylglutaryl coenzyme A reductase. I. Biol. Chem. 266: 13947-13951.

Osborne, T.F., G. Gil, J.L. Goldstein, and M.S. Brown. 1988. Operator constitutive mutation of 3-hydroxy-3-methylglutaryl coenzyme A reductase promoter abolishes protein binding to sterol regulatory element. J. Biol. Chem. 263: 3380-3387.

Panini, S.R., R.J. Lutz, L. Wenger, J. Miyake, S. Leonard, A. Andalibi, A.J. Lusis, and M. Sinensky. 1990. Defective elongation of fatty acids in a recessive 25-hydroxycholesterolresistant mutant cell line. J. Biol. Chem. 265: 14118-14126.

Peffley, D., J. Miyake, S. Leonard, C. von Gunten, and M. Sinensky. 1988. Further characterization of a somatic cell mutant defective in regulation of 3-hydroxy-3-methylglutaryl coenzyme A reductase. Somatic Cell Mol. Genet. 14: 527539.

Porges, A.J., T. Ng, and W.H. Reeves. 1990. Antigenic determinants of the $\mathrm{Ku}(\mathrm{p} 70 / \mathrm{p} 80)$ autoantigen are poorly conserved between species. J. Immunol. 145: 4222-4228.

Reeves, W.H. and Z.M. Sthoeger. 1989. Molecular cloning of cDNA encoding the p70 (Ku) lupus autoantigen. I. Biol. Chem. 264: 5047-5052.

Sambrook, J., E.F. Fritsch, and T. Maniatis. 1989. Molecular cloning: A laboratory manual. Cold Spring Harbor Laboratory Press, Cold Spring Harbor, New York.

Sato, R., J. Yang, X. Wang, M.J. Evans, Y.K. Ho, J.L. Goldstein, and M.S. Brown. 1994. Assignment of the membrane attachment, DNA binding, and transcriptional activation domains of sterol regulatory element binding protein-1 (SREBP-1). $J$. Biol. Chem. 25: 17267-17273.

Sinensky, M., G. Duwe, and F. Pinkerton. 1979. Defective regulation of 3-hydroxy-3-methylglutaryl coenzyme A reductase in a somatic cell mutant. J. Biol. Chem. 254: 4482-4486.

Wang, X., M.R. Briggs, X. Hua, C. Yokoyama, J.L. Goldstein, and M.S. Brown. 1993. Nuclear protein that binds sterol regulatory element of LDL receptor promoter II. Purification and Characterization. J. Biol. Chem. 268: 14497-14504.
Wang, X., R. Sato, M.S. Brown, X. Hua, and J.L. Goldstein. 1994 SREBP-1, a membrane-bound transcription factor released by sterol-regulated proteolysis. Cell 77: 53-62.

Yokoyama, C., X. Wang, M.R. Briggs, A. Admon, J. Wu, X. Hua, J.L. Goldstein, and M.S. Brown. 1993. SREBP-1, a basic helixloop-helix leucine zipper protein that controls transcription of the LDL receptor gene. Cell 75: 187-197. 


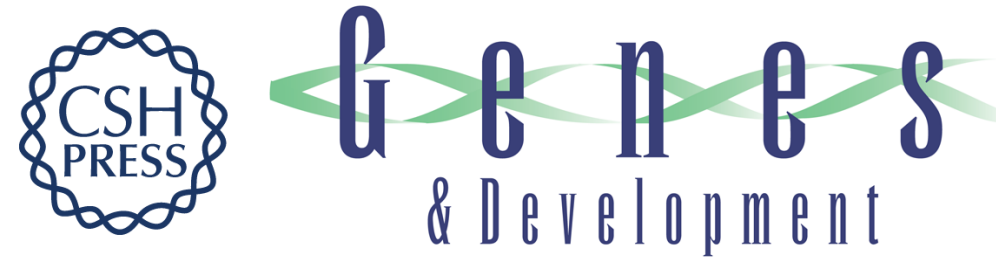

\section{Sterol-resistant transcription in $\mathrm{CHO}$ cells caused by gene rearrangement that truncates SREBP-2.}

$J$ Yang, R Sato, J L Goldstein, et al.

Genes Dev. 1994, 8:

Access the most recent version at doi:10.1101/gad.8.16.1910

References This article cites 23 articles, 13 of which can be accessed free at:

http://genesdev.cshlp.org/content/8/16/1910.full.html\#ref-list-1

License

Email Alerting Receive free email alerts when new articles cite this article - sign up in the box at the top Service right corner of the article or click here.

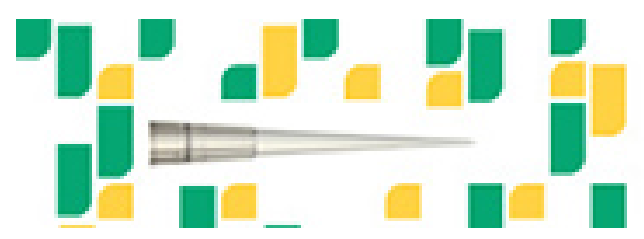

Focused on your science.

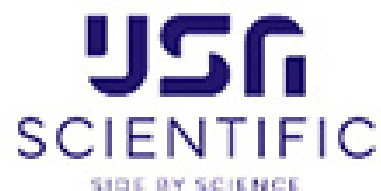

Copyright (c) Cold Spring Harbor Laboratory Press 